\title{
HEAT TRANSFER MODEL FOR AN OPEN-CYCLE GAS CORE NUCLEAR ROCKET
}

\author{
David I. Poston and Terry Kammash \\ Dept. of Nuclear Engineering \\ University of Michigan \\ Ann Arbor, MI 48109 \\ (313) 747-0900
}

\begin{abstract}
A heat transfer model is developed to assess the propulsion capability of the open-cycle gas core nuclear rocket. The model is used to determine the maximum specific impulse achievable without violating the wall material temperature and heat flux limits. For a $3000 \mathrm{MW}$ reactor with a wall heat flux limit of $100 \mathrm{MW} / \mathrm{m}^{2}$, it is shown that a specific impulse of $3160 \mathrm{~s}$ and a thrust of $125 \mathrm{kN}$ can be obtained.
\end{abstract}

\section{INTRODUCTION}

The Gas Core Nuclear Rocket (GCR) is a propulsion scheme that could allow manned missions to Mars in relatively short times. In the GCR a fissioning uranium plasma heats (primarily by radiation) a hydrogen propellant, which is exhausted through a choked nozzle. In this paper we carry out a heat transfer analysis to determine the important propulsion parameters, namely specific impulse and thrust, and establish their dependence on various parameters and limitations of the system.

The propulsion capability of the rocket is dependent on the state of the propellant in the reservoir proceeding the nozzle throat. A computer code was written to calculate the propellant temperature in a high power density nuclear rocket as a function of power, dimensions, mass flow rate, and initial conditions. The propellant (hydrogen) flows with a constant mass flow rate through a cylindrical annulus surrounding the reactor core. The code offers the option of including the fuel region (uranium) in the heat transfer model (in which the power is a function of the fuel density), or simply specifying a heat flux at the fuel/propellant interface. In both cases it is assumed that the fuel and propellant do not mix. The outer cavity wall is assumed to remain at a constant temperature due to transpiration cooling.

The thermal radiation absorption coefficient of the propellant is sufficiently high so that a diffusion heat transfer analysis can be used (the absorption cross section of hydrogen is relatively low at temperatures less than $10,000 \mathrm{~K}$, thus the hydrogen is seeded with solid particles to aid in the absorption of radiation at low temperatures). The diffusion method models radiative heat transfer in the form of conduction, with a conduction coefficient depending on $\mathrm{T}^{3}$.

The code uses an iterative finite difference scheme to calculate propellent temperatures as a function of radial and axial position. An iterative solution is required because the radiative 'conductivity' coefficient is temperature dependent, making the set of heat transfer equations non-linear. The results of these calculations are used to evaluate rocket performance for various reactor designs.

\section{ANALYSIS}

\section{Temperature Solution}

The fundamental conservation equations of mass, momentum, and energy are solved numerically.

$$
\begin{gathered}
\frac{\partial \rho}{\partial t}+\nabla \cdot \rho Y=0, \\
\rho \frac{D \underline{\underline{Y}}}{D t}=-\nabla P-\nabla \cdot \underline{\underline{I}}+\rho \mathcal{g}, \text { and }
\end{gathered}
$$




$$
\rho C_{p} \frac{D T}{D t}=-(\nabla q)+\frac{D P}{D t}-(\tau: \nabla v)+Q_{\nu}
$$

The continuity equation is used to determine the flow velocity. The momentum equation is nullified by the following assumptions: steady inviscid flow, constant pressure, and no external forces. To simplify the energy equation, it is also assumed that: fluid properties do not change significantly over one mesh interval, there is no axial conduction, and that azimuthal symmetry applies. The steady state energy equation becomes:

$$
\rho C_{p}\left(v_{z} \frac{\partial T}{\partial z}+v_{r} \frac{\partial T}{\partial r}\right)=-\frac{1}{r} \frac{\partial}{\partial r}\left(r q_{r}\right)+Q_{v} .
$$

The volumetric power generation $Q_{v}$ is dependent on the fuel density, and the radial velocity $v_{r}$ is assumed to be zero except when transpiration cooling is required. Using the diffusion method of heat transfer:

$$
q_{r}=-k \frac{\partial T}{\partial r} \quad\left(k=k_{\text {cond }}+k_{\text {rod }}=k_{c}+\frac{16 \sigma T^{3}}{3 a_{r}}\right),
$$

where $a_{r}$ is the Rosseland mean absorption coefficient. The value of $a_{r}$ includes the absorptivity of solid seed particles inserted in the flow to aid radiation absorption at low temperatures.

The diffusion approximation is valid in all regions except near the wall, where the optical thickness is low. An exponential absorption integral is evaluated to more accurately predict wall heat flux:

$$
q_{\text {wall }}=\frac{1}{r_{w}} \int_{r_{w}}^{r_{w}} r a_{p} \sigma T^{4} e^{-\int_{r} a_{p} d r^{\prime}} d r+\frac{r_{\operatorname{lnt}}}{r_{w}} q_{i n t} e^{-\int_{r}^{r_{w}} a_{p} d r^{\prime}} .
$$

The conductivity coefficient at the wall is iterated until the diffusion solution produces a wall heat flux consistent with the absorption integral.

The above equations are solved by the method of finite differencing. The boundary conditions are determined by the wall temperature, and either the fuel/propellant interface heat flux (if the fuel region is not modelled, or symmetry at the centerline (if the fuel region is modelled). An iterative solution is required because the fluid properties and the radiative conduction coefficient are temperature dependent.

If a maximum wall heat flux is specified, then the boundary condition at the outer wall changes. In addition to specifying the wall temperature $T_{w}$, the temperature at the node adjacent to the wall is limited to a maximum value of:

$$
T_{\text {cool }}=T_{w}+\frac{\Delta r q_{\max }}{2 k_{w}} .
$$

The transpiration cooling flow rate required to maintain this temperature is found via the energy equation. It is assumed that the cooling fluid (also hydrogen) is instantaneously heated from $T_{w}$ to $T_{\text {cool }}$, and that the cooling fluid does not interact with the principle flow (the flow entering through the core inlet) until it reaches the reservoir.

In order to evaluate rocket performance, the reservoir temperature must be determined. The reservoir is considered to be the region between the core exit and the nozzle throat. Here it is assumed that the propellant (including the cooling fluid) comes to a uniform temperature, which is determined by the average enthalpy of the fluid.

\section{Rocket Performance}

To calculate rocket performance the core exit region is modelled as a reservoir (the kinetic energy of the fluid is negligible) from which the propellant flows through a chocked nozzle to a vacuum. The nozzle expansion ratio is defined by user input. The thrust and specific impulse are both a function of the equivalent velocity. 


$$
\begin{gathered}
F=\dot{m} v_{e q} \quad \text { and } \quad I_{p}=\frac{v_{e q}}{g} \\
\text { where: } v_{e q}=v_{e}+\frac{P_{e} A_{e}}{\dot{m}} .
\end{gathered}
$$

Therefore the exit velocity, pressure, and area must be calculated.

Modelling the propellant flow through the nozzle is difficult since the fluid may dissociate, ionize and/or recombine. As a simple approach, the standard isentropic compressible flow equations are used, which yi $1 \mathrm{l}$ $T, P$, and $\rho$ downstream in terms of $M$. The exit velocity and area are (note that $C_{p}=\gamma R /[\gamma-1]$ ):

$$
\begin{aligned}
v_{e}=M_{e} \sqrt{\gamma R_{e} T_{e}} & =M_{e} \sqrt{(\gamma-1) C_{p_{e} T_{e}}} \text {, and } \\
A_{e} & =\frac{\dot{m}}{\rho_{e} \nu_{e}} .
\end{aligned}
$$

It was found that this approach yields values of $\mathrm{I}_{\text {sp }} 10 \%$ to $20 \%$ lower than calculated by Patch (1971), in which the flow of high temperature hydrogen through a choked nozzle is modelled much more thoroughly. Therefore, it was decided to use values of thrust and specific impulse based on data from Patch (1971), rather than using the above equations. However the above approach does provide a means of estimating the effect of uranium flow on rocket performance. The specific impulse of multi-species flow is:

$$
I_{s p}=\frac{1}{g} \frac{\sum_{i} \dot{m}_{i} v_{e q_{1}}}{\sum_{i} \dot{m}_{i}} .
$$

\section{Fluid Properties}

Fluid properties were obtained from various reports. For hydrogen the specific heat and density were taken from Patch (1971), while the thermal conductivity (which is important only at low temperatures) was found in Incropera and DeWitt (1981), and the Rosseland and Planck mean absorptivities were taken from Patch (1969). All of the required data for uranium was given in Parks et al. (1968). The hydrogen is assumed to contain .7 weight percent seed material with an absorption cross section of $5000 \mathrm{~m}^{2} / \mathrm{kg}$. This value was experimentally achieved by Williams et al. (1969).

\section{RESULTS AND DISCUSSION}

\section{Temperature Solution}

The input parameters of a large GCR, similar to one referenced by Borowski (1987), are listed below along with the corresponding results.

\section{Input Parameters}

Reactor Power

Reactor Pressure

Max. Wall Heat Flux

Inlet Prop. Flow Rate

Inlet Temperature

Wall Temperature

Fuel/Prop. Flow Ratio

Core Length

Fuel Region Radius

Outer Wall Radius

\section{$\underline{\text { Results }}$}

Fuel reservoir temp.

Prop. reservoir temp.

Wall cooling flow

$66320 \mathrm{~K}$

$1000 \mathrm{~atm}$

$100 \mathrm{MW} / \mathrm{m}^{2}$

$3.7 \mathrm{~kg} / \mathrm{s}$

$2200 \mathrm{~K}$

$2200 \mathrm{~K}$

$10 / 1$

$2.0 \mathrm{~m}$

$0.8 \mathrm{~m}$

$1.0 \mathrm{~m}$
Specific Impulse $3160 \mathrm{~s}$

Thrust
$17880 \mathrm{~K}$

$0.4 \mathrm{~kg} / \mathrm{s}$

$125 \mathrm{kN}$

Figures 1 and 2 contain the axial and radial temperature distributions for the case listed above. 


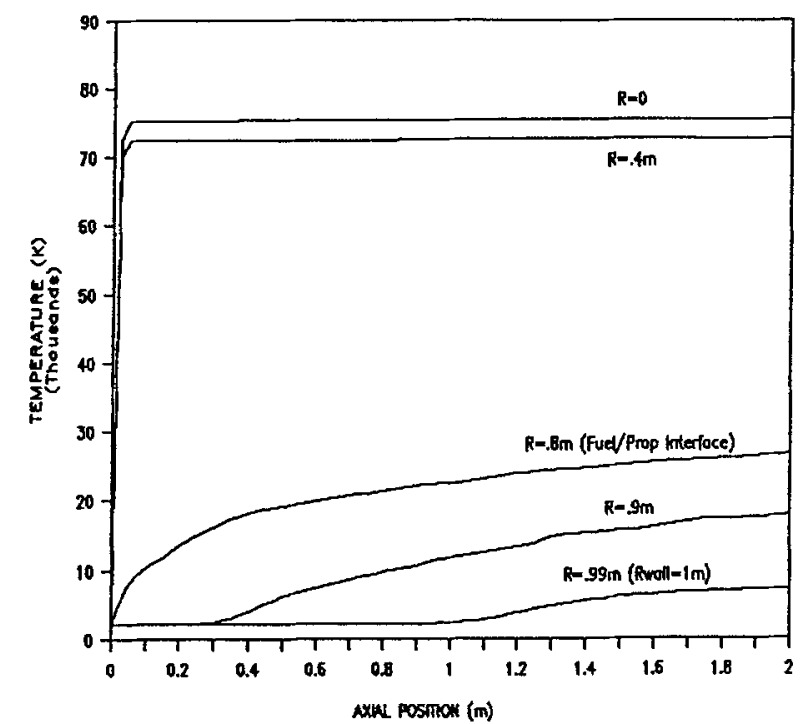

FIGURE 1. Axial Temperature Profile.

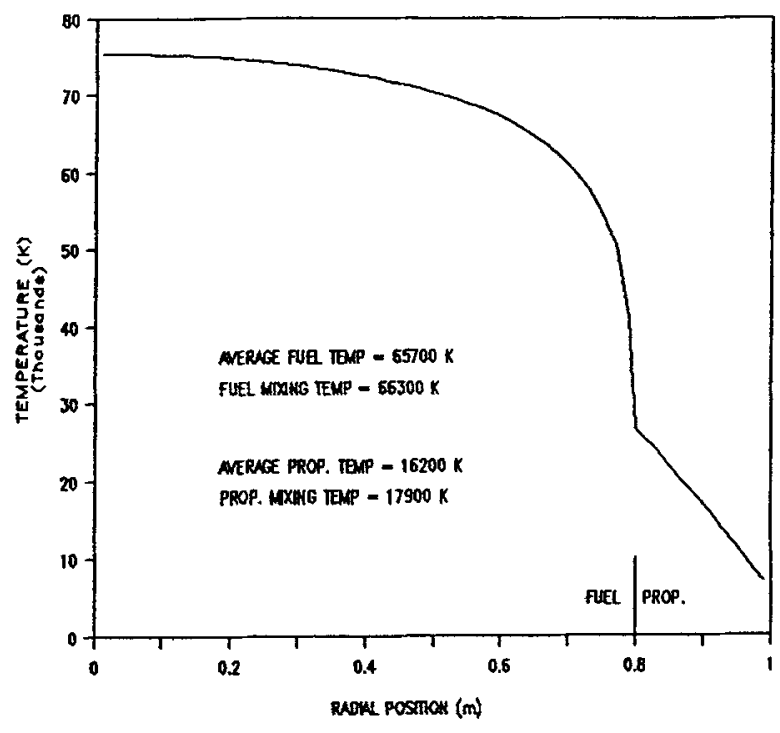

FIGURE 2. Radial Temp. Profile at Core Exit.

In Figure 1 it can be seen that the fuel reaches its equilibrium temperature very quickly. This is due to the low velocity and the relatively low specific heat of the uranium. Once the fuel reaches its equilibrium temperature profile, all of the energy generated in the fuel is transferred to the propellant. Therefore the fuel region temperature and interface heat flux are essentially constant, except at locations very near the core inlet. Consequently there is almost no difference between modelling the fuel region, or simply specifying the fuel/propellant interface heat flux. So unless fuel temperatures are desired, it is more convenient to specify the interface heat flux and ignore the fuel region.

Since the fuel quickly reaches its equilibrium temperature, the overall thermal conductivity between the fuel and propellant does not significantly impact the propellant temperature. If the conductivity is reduced, then the fuel temperature will simply increase to a temperature which will produce the same interface heat flux. Therefore if fuel/propellant mixing were to be modelled, the increase of the heat transfer coefficient at the interface would not significantly change the results. This does not diminish the overall significance of fuel/propellant mixing, which is of primary importance when calculating containment and criticality conditions.

\section{Rocket Performance}

The effects of various reactor parameters on rocket performance are discussed below.

Inlet Mass Flow Rate - Figure 3 shows how specific impulse varies with the inlet mass flow rate. If there is no maximum wall heat flux specified, the specific impulse increases as the flow rate decreases until a maximum of $4000 \mathrm{~s}$ is reached. This maximum indicates the propellant has reached an equilibrium temperature, meaning that any additional energy generated in the fuel will be conducted to the wall. If a maximum wall heat flux is specified, then the specific impulse reaches a peak at some optimum flow rate. If the inlet flow is reduced below this value then transpiration cooling is required, thus lowering the specific impulse.

Max. Wall Heat Flux - The specific impulse and thrust of the reactor described above are plotted versus maximum wall heat flux on Figure 4. As $q_{\max }$ is increased the maximum obtainable $I_{s p}$ increases until it reaches a value of $4000 \mathrm{~s}$, this indicates that the propellant has reached its equilibrium temperature. At low values of $q_{\max }$ the $I_{s p}$ levels off around $2200 \mathrm{~s}$. This value of $I_{s p}$ can be obtained without any heat reaching the outer wall. 


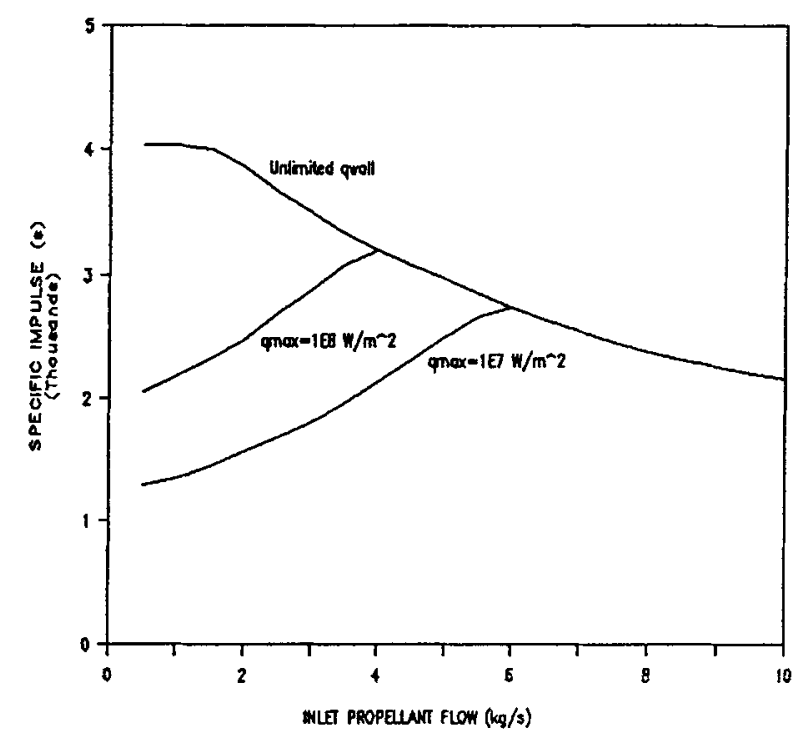

FIGURE 3. $I_{\mathrm{sp}}$ Dependence on Inlet Flow Rate.

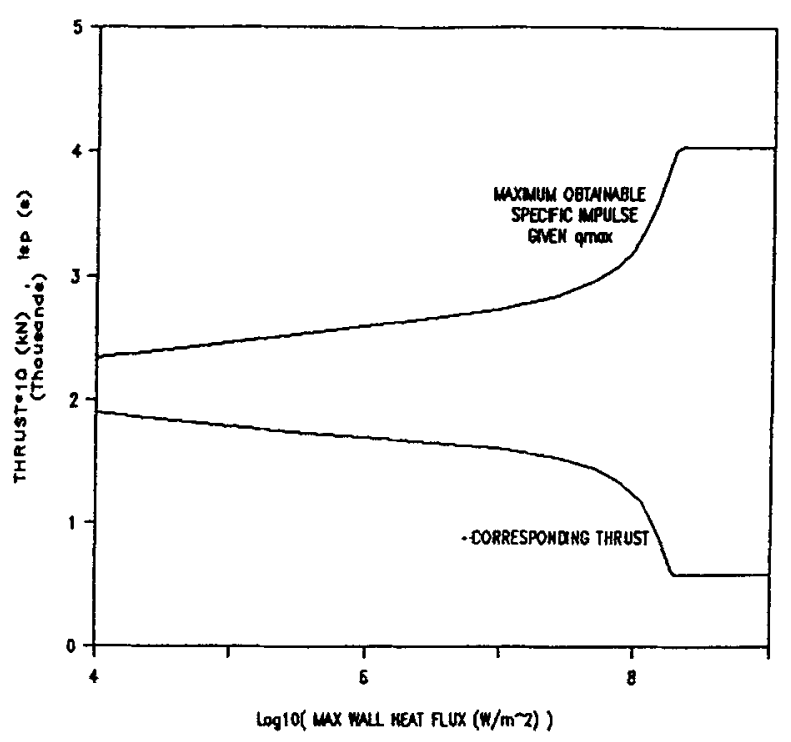

FIGURE 4. $I_{\text {sp }}$ Dependence on Max. Wall Heat Flux.

Dimensions - The dimensions of the system have little effect on the propellant temperature, and thus specific impulse. This is also due to the fact that nearly all of the energy generated flows to the propellant. However, increasing the dimensions can help reduce the wall heat flux by increasing the wall surface area. Yet this benefit may be nullified by the resulting mass penalty.

Power - Increased reactor power improves overall rocket performance, but not necessarily specific impulse. If reactor power is increased, the flow rate must be increased to avoid exceeding the critical wall heat flux, resulting in the same specific impulse but higher thrust. Likewise if power is reduced, the flow rate can be reduced, yielding the same specific impulse but lower thrust.

Uranium/Seed Particle Flow - Using the standard isentropic compressible flow equations, the effect of uranium flow on rocket performance was estimated. For a 10/1 propellant to fuel mass flow ratio, the specific impulse decreased $6 \%$ and thrust increased $2 \%$. This decrease in specific impulse is relatively small because the uranium is at such a high temperature $(\sim 66,000 \mathrm{~K})$. The seed particle amounts to only $.7 \%$ of the propellant mass, thus should not significantly effect rocket performance.

Inlet/Wall Temperature - The temperature of the inlet fluid and wall has a minor effect on rocket performance. An increase in the wall and inlet temperature from $1100 \mathrm{~K}$ to $2200 \mathrm{~K}$ causes less than a $1 \%$ increase in $I_{s p}$ and thrust. Possibly more important than the increase in performance, is the increase in the regenerative capacity of the propellant before entering the core. Thus eliminating the need for, or reducing the size of a possible radiator.

\section{CONCLUSIONS}

A heat transfer model is developed to assess the propulsion capability of the open-cycle gas core nuclear rocket. Using this model the open-cycle GCR is found to yield a very attractive combination of thrust and specific impulse. The major limiting factor in achieving high specific impulse is the maximum wall heat flux. The results of this and other studies indicate that although there are several major technical issues that must be addressed, the open-cycle gas core nuclear rocket merits future consideration as a high performance propulsion scheme.. 


\section{Acknowledgments}

All work was done at the University of Michigan. The work was sponsored by NASA Lewis Research Center as part of the NASA Graduate Researchers Program. The authors would like to acknowledge John Clark and Stan Borowski of NASA LeRC for their assistance.

\section{References}

Borowski, S. K. (1987) Nuclear Propulsion - A Vital Technology for the Exploration of Mars and the Planets Beyond, NASA TM-101354, NASA/Lewis Research Center, Cleveland, $\mathrm{OH}$.

Incropera F. R. and D. P. DeWitt (1981) Fundamentals of Heat Transfer, John Wiley and Sons, New York, pp. 777-778.

Parks, D. E. et al. (1968) Optical Constants of Uranium Plasma, NASA CR-72348, Gulf General Atomic, San Diego, CA.

Patch, R. W. (1969) Interim Absorption Coefficients and Opacities for Hydrogen at High Pressure, NASA TM X-1902, NASA/Lewis Research Center, Cleveland, $\mathrm{OH}$.

Patch, R. W. (1971) Thermodynamic Properties and Theoretical Performance of Hydrogen to $100000 \mathrm{~K}$ and $1.01325 \times 10^{8} \mathrm{~N} / \mathrm{m}^{2}$, NASA SP-3069, NASA/Lewis Research Center, Cleveland, $\mathrm{OH}$.

Williams, J. R., J. D. Clement, A. S. Shenoy, W. L. Partain (1969) The Attenuation of Radiant Energy in Hot Seeded Hydrogen, Quarterly Status Report 2, Georgia Institute of Technology, Atlanta, GA.

\section{Nomenclature}

English

A: $\quad$ Flow area $(m)$

$a_{p}: \quad$ Planck mean absorptivity $(1 / \mathrm{m})$

$a_{r}$ : $\quad$ Rosseland mean absorptivity $(1 / \mathrm{m})$

$\mathrm{C}_{\mathrm{p}}: \quad$ Specific Heat $(\mathrm{J} / \mathrm{kg} \cdot \mathrm{K})$

F: $\quad$ Thrust (N)

g: $\quad$ Gravitational constant $(9.81 \mathrm{~m} / \mathrm{s})$

h: $\quad$ Enthalpy $(\mathrm{J} / \mathrm{kg})$

$\mathrm{I}_{\mathrm{sp}}$ : $\quad$ Specific impulse (s)

$\mathrm{k}$ : Thermal conductivity $(\mathrm{W} / \mathrm{m} \cdot \mathrm{K})$

M: $\quad$ Mach number

m: $\quad$ Mass flow rate $(\mathrm{kg} / \mathrm{s})$

P: $\quad$ Pressure $(\mathrm{Pa})$

$\mathrm{q}: \quad$ Heat flux $\left(\mathrm{W} / \mathrm{m}^{2}\right)$

$\mathrm{Q}_{\mathrm{v}}: \quad$ Volumetric heat generation $\left(\mathrm{W} / \mathrm{m}^{3}\right)$

r: $\quad$ Radial position (m)

T: $\quad$ Temperature (K)

v: $\quad$ Flow velocity $(\mathrm{m} / \mathrm{s})$

z: Axial position (m)

\section{Greek}

$\gamma: \quad$ Ratio of specific heats

p: Density $\left(\mathrm{kg} / \mathrm{m}^{3}\right)$

б: Stefan-Boltzmann constant $\left(5.67 \times 10^{-8} \mathrm{~W} / \mathrm{m}^{2} \cdot \mathrm{K}^{4}\right)$

$\tau$ : $\quad$ Stress tensor

\section{Subscripts}

e: Nozzle exit

in: Reactor inlet

int: Fuel/prop interface

w: Reactor wall 